\title{
Venous thromboembolism and gastrointestinal stromal tumour: A rare association
}

\author{
FRANCISCO GALEANO-VALLE ${ }^{1,2}$, JORGE DEL-TORO-CERVERA ${ }^{1,2}$ and PABLO DEMELO-RODRIGUEZ ${ }^{1,2}$ \\ ${ }^{1}$ Venous Thromboembolism Unit, Internal Medicine Department, University Hospital Gregorio Marañón; \\ ${ }^{2}$ Sanitary Research Institute Gregorio Marañón, Madrid 28007, Spain
}

Received January 15, 2019; Accepted August 1, 2019

DOI: $10.3892 / \mathrm{mco} .2019 .1942$

\begin{abstract}
Gastrointestinal stromal tumours (GISTs) comprise $0.1-3 \%$ of all gastrointestinal malignancies and represent the majority of gastrointestinal mesenchymal neoplasms. The major cause of GIST is an abnormal form of tyrosine kinase protein. GISTs may arise anywhere in the gastrointestinal tract, but are more commonly encountered in the stomach and small intestine. Gastrointestinal bleeding and abdominal pain are the most frequent symptoms associated with gastric GISTs. However, venous thromboembolism (VTE) is an uncommon presentation of these tumours. To the best of our knowledge, only 4 cases highlighting the association between these rare tumours and VTE have been reported in the literature to date. We herein report a case of a gastric GIST presenting with pulmonary embolism. Current guidelines recommend anticoagulation with low-molecular-weight heparin as first-line treatment for the acute phase of cancer-associated thrombosis. Prolonged anticoagulation should be considered for an indefinite time period, or until curative treatment. In the present case, tumour resection enabled a reduction in the duration of the anticoagulation treatment, thereby reducing the risk of long-term bleeding complications.
\end{abstract}

\section{Introduction}

Gastrointestinal stromal tumours (GISTs) comprise $0.1-3 \%$ of all gastrointestinal malignancies and represent the majority of the gastrointestinal mesenchymal neoplasms. The major cause of GIST is an abnormal form of tyrosine kinase protein. GISTs may arise anywhere in the gastrointestinal tract, but are more commonly detected in the stomach and small intestine. Gastrointestinal bleeding and abdominal pain are the most

Correspondence to: Dr Francisco Galeano-Valle, Venous Thromboembolism Unit, Internal Medicine Department, University Hospital Gregorio Marañón, 46 Calle del Doctor Esquerdo, Madrid 28007, Spain

E-mail: paco.galeano.valle@gmail.com

Key words: anticoagulation, deep venous thrombosis, gastrointestinal stromal tumour, pulmonary embolism, venous thromboembolism frequent symptoms associated with gastric GISTs. Surgical resection currently remains the gold standard in the treatment of GISTs, and it is indicated even when incomplete and aimed at symptom palliation. Unresectable GISTs should be managed by molecularly targeted intervention with imatinib mesylate (1).

Venous thromboembolism (VTE) is a common complication in cancer patients, and three basic underlying mechanisms have been described: Decreased blood flow, injury to the vessel wall and disturbances in the balance between procoagulant and anticoagulant factors (2). However, the association and the pathophysiological mechanisms between GISTs and VTE remain unclear. To the best of our knowledge, there are only 4 cases in the currently available literature highlighting the association between these rare tumours and VTE (2-5).

We herein present a case of a gastric GIST presenting with pulmonary embolism and discuss the mechanisms implicated in the pathogenesis and the management of this disease.

\section{Case report}

The patient was an 87-year-old woman with a past history of secondary hypothyroidism five years following thyroidectomy for non-toxic multinodular goiter, and no previous history of VTE. The patient had no recent history of immobilization, surgery or prolonged travel. She had no varicose veins in the lower limbs and her body mass index was $28.2 \mathrm{~kg} / \mathrm{m}^{2}$. The patient reported weight loss and progressive cachexia over the last 2 months.

The patient presented in May 2017 to the Emergency Department of University Hospital Gregorio Marañón with a sudden onset of chest pain without dyspnea, fever or cough. On physical examination, her blood pressure was $150 / 80 \mathrm{mmHg}$, with a heart rate of $103 \mathrm{bpm}$. The remaining findings on physical examination were unremarkable. The findingsofthebloodanalysis were as follows: Haemoglobin $12.5 \mathrm{~g} / \mathrm{dl}, 215,000$ platelets $/ \mathrm{mm}^{3}$, creatinine $0.67 \mathrm{mg} / \mathrm{dl}$, D-dimer 2,350 $\mathrm{ng} / \mathrm{ml}$ and Nt-proBNP $378 \mathrm{pg} / \mathrm{ml}, \mathrm{pO}_{2} 52 \mathrm{~mm} \mathrm{Hg}$ and $\mathrm{pCO}_{2} 38 \mathrm{~mm} \mathrm{Hg}$. A computed tomography pulmonary angiography revealed multiple bilateral filling defects in segmental and subsegmental arteries (Fig. 1A), and weight-adjusted enoxaparin was initiated.

An abdominal computed tomography revealed a solid heterogeneous $5.8 \times 3.5 \times 3.5 \mathrm{~cm}$ submucous mass in the gastric fundus, suggesting the presence of a GIST (Fig. 1B). Upper 

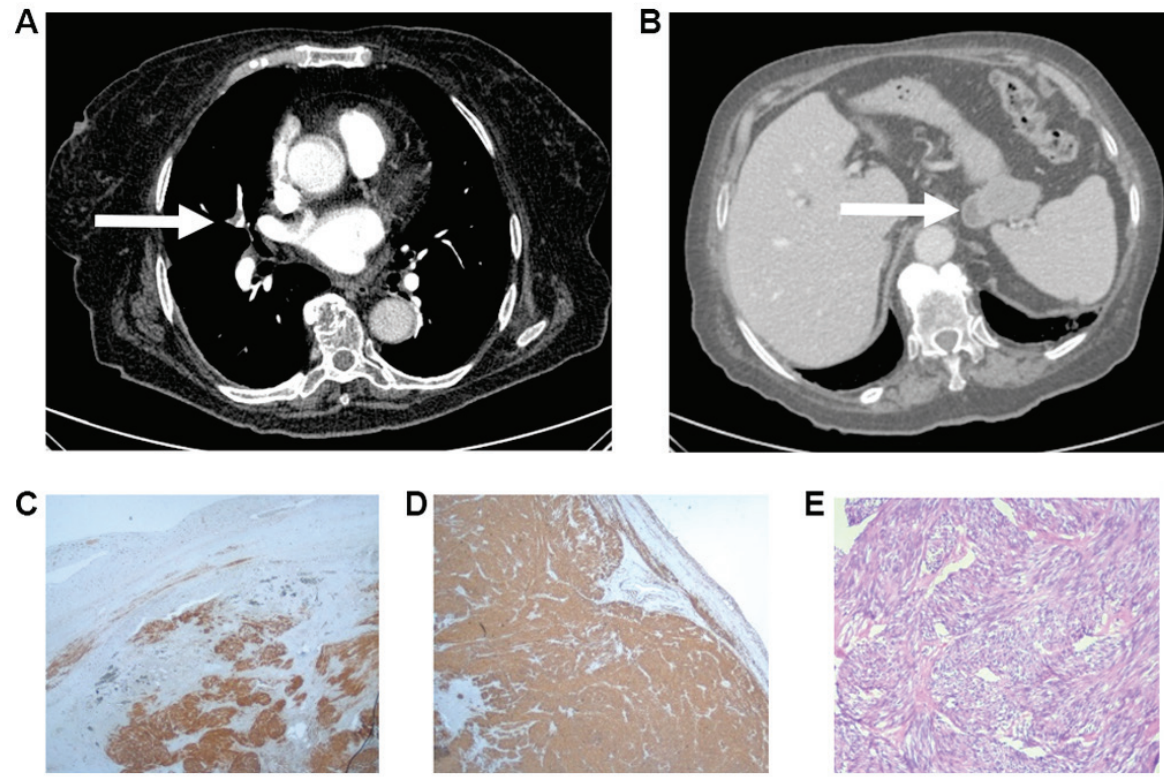

Figure 1. (A) A computed tomography pulmonary angiography revealed multiple filling defects in segmental and subsegmental arteries of the right upper, middle and lower lobes (arrow). (B) An abdominal computed tomography revealed a solid heterogeneous mass, sized 5.8x3.5x3.5 cm, in the submucous layer of the gastric fundus (arrow), resembling a gastrointestinal stromal tumour (GIST). Immunohistochemical staining for (C) C-KIT and (D) CD34 revealed strong and diffuse cytoplasmic expression (original magnification, x200). (E) Spindle-cell GIST composed of fascicles of uniform cells with pale eosinophilic cytoplasm (haematoxylin-eosin staining; original magnification, $\mathrm{x} 400$ ).

endoscopy revealed a submucosal lesion in gastric fundus. A Doppler-ultrasound of the lower extremities ruled out deep vein thrombosis (DVT), and the findings on echocardiography were normal. The patient was discharged with enoxaparin $100 \mathrm{mg} /$ day. Three months later, the patient underwent a gastric wedge resection and the sample was sent for histopathological examination (Fig. 1C-E). The patient was treated with enoxaparin $100 \mathrm{mg} /$ day for 3 months. Then, enoxaparin was discontinued one day before the surgery, and initiated again at $24 \mathrm{~h}$ after the surgery. A haematoma in the right flank appeared on the second postoperative day, requiring enoxaparin withdrawal for 5 days. Anticoagulation was discontinued 3 months after surgery. Last follow up was in December 2018 and the patient has remained asymptomatic and has not showed evidence of recurrence of venous thrombosis or cancer.

\section{Discussion}

VTE is a common complication in cancer patients and three basic mechanisms have been suggested: Decreased blood flow, injury to the vessel wall and disturbances in the balance between procoagulant and anticoagulant factors (2). However, the association and pathophysiological mechanisms between GISTs and VTE remain unclear. To the best of our knowledge, there are only 4 cases in the literature highlighting the association between these rare tumours and VTE. Melichar et al presented the case of a patient with peritoneal metastases of GIST involving the pelvis who was treated with imatinib and succumbed to pulmonary embolism (PE) and DVT caused by the compression of the common iliac vein by the tumour (2). Kardos et al reported a case of gastric GIST presenting as DVT and PE. In that case, thorough evaluation did not reveal an aetiology for thrombosis other than the GIST, suggesting that the neoplasm was the primary cause (3). Sam et al presented the case of a patient with small bowel GIST who was diagnosed after jejunal intussusception and developed an episode of DVT during hospitalization (4). Thus, it may be hypothesized that the episode was cause by surgery and immobilization, in addition to the presence of the tumour. Finally, our group recently published the case of a patient with a $18 \times 12 \mathrm{~mm}$ gastric GIST managed conservatively with close endoscopic follow-up, who developed an episode of PE 6 months after the diagnosis. In that case, the neoplasm was the only provoking factor identified. The patient underwent tumour resection, allowing a reduction in the duration of anticoagulant therapy (5). According to the recommendations of the European Society of Medical Oncology-European Reference Network for Rare Adult Solid Cancers, the standard approach to tumours $>2 \mathrm{~cm}$ in size is biopsy/excision, as they are associated with a higher risk of progression if confirmed as GIST. Then, the standard treatment for localized GISTs is complete surgical excision, without dissection of clinically negative lymph nodes (6). The importance of the present case report is that an associated VTE episode may worsen the prognosis of the tumour, as VTE is a major cause of death in patients with any type of cancer. Therefore, it is crucial to highlight the association between VTE and this rare type of cancer, as confirmation of such an association may prompt more aggressive management of patients with GISTs.

The current guidelines recommend anticoagulation with low-molecular-weight heparin as first-line treatment for the acute phase of cancer-associated VTE. Prolonged anticoagulation should be considered for an indefinite period of time or until curative treatment (7). In the present case, tumour resection enabled a reduction in the duration of the anticoagulation, thereby reducing the risk of long-term bleeding complications.

In summary, we herein present a case of GIST with associated VTE. Since the association between these two entities has not been clearly stablished, further studies 
evaluating the frequency of VTE in patients with GIST are required.

\section{Acknowledgements}

Not applicable.

\section{Funding}

No funding was received.

\section{Availability of data and materials}

Not applicable.

\section{Authors' contributions}

FGV, JDTC and PDR contributed to the conception and design of the study, provided administrative support and study materials, and recruited patients. Furthermore, they collected and analysed data, as well as wrote the manuscript. All authors have read and approved the final version of this manuscript for publication.

\section{Ethics approval and consent to participate}

The study was approved by the Research Ethics Committee of the University Hospital Gregorio Marañón.

\section{Patient consent for publication}

The patient provided written informed consent regarding the publication of the case details and any associated images.

\section{Competing interests}

The authors declare that they have no competing interests.

\section{References}

1. El-Menyar A, Mekkodathil A and Al-Thani H: Diagnosis and management of gastrointestinal stromal tumours: An up-to-date literature review. J Can Res Ther 13: 889-900, 2017.

2. Melichar B, Laco J, Slovácek L, Grossmann P and Vanecek T: Fatal venous thrombembolism complicating imatinib therapy in a patient with metastatic gastrointestinal stromal tumour. J Exp Clin Cancer Res 25: 607-610, 2006.

3. Kardos M, Lundquist A, Misialek M and Friedman LS: A rare presentation of an uncommon malignancy: Thromboembolism in a patient with a gastrointestinal stromal tumour. Dig Dis Sci 56: 2711-2714, 2011

4. Sam JJ, Mustard R, Kandel G, Gardiner G, Ghaffar H, Kirpalani A, May G and Kim YI: Colonoscopy leads to a diagnosis of a jejunal gastrointestinal stromal tumour (GIST). Gastroenterology Res 4: 277-282, 2011.

5. Demelo-Rodríguez P, Lavilla Olleros C, Martín Higueras E, Peligros I and del Toro-Cervera J: Tumour del estroma gastrointestinal como causa de trombosis asociada a cáncer. Rev Gastroenterol Méx 84: 250-252, 2019.

6. Casali PG, Abecassis N, Aro HT, Bauer S, Biagini R, Bielack S, Bonvalot S, Boukovinas I, Bovee JVMG, Brodowicz T, et al: Gastrointestinal stromal tumours: ESMO-EURACAN clinical practice guidelines for diagnosis, treatment and follow-up. Ann Oncol 29 (Suppl 4): iv267, 2018.

7. Konstantinides SV, Torbicki A, Agnelli G, Danchin N, Fitzmaurice D, Galiè N, Gibbs JS, Huisman MV, Humbert M, Kucher N, et al: Task force for the diagnosis and management of acute pulmonary embolism of the european society of cardiology (ESC). 2014 ESC guidelines on the diagnosis and management of acute pulmonary embolism. Eur Heart J 35: 3033-3069, 2014. 